\title{
Advanced Mirror Technology Development
}

\author{
H. Philip Stahl \\ NASA Marshall Space Flight Center, Huntsville Alabama 35821 \\ h.philip.stahl@nasa.gov
}

\begin{abstract}
The Advanced Mirror Technology Development (AMTD) project matures critical technologies required to enable ultra-stable 4-m-or-larger monolithic or segmented ultraviolet, optical, and infrared (UVOIR) space telescope primary-mirror assemblies for general astrophysics and ultra-high-contrast observations of exoplanets.

OCIS codes: (220.4880) Optomechanics; (220.4610) Optical Fabrication; (350.1260) Astronomical Optics
\end{abstract}

\section{Introduction}

The Advanced Mirror Technology Development (AMTD) project is a multiyear effort initiated in 2012, to mature critical technologies required to enable 4-m-or-larger ultraviolet, optical, and infrared (UVOIR) space telescope primary-mirror assemblies for general astrophysics and ultra-high-contrast observations of exoplanets. UVOIR measurements provide robust, often unique, diagnostics for investigating astronomical environments and objects. UVOIR observations are responsible for much of our current astrophysics knowledge and will produce as-yetunimagined, paradigm-shifting discoveries. A new, larger UVOIR telescope is needed to help answer fundamental scientific questions such as: Does life exist on nearby Earth-like exoplanets? How do galaxies assemble their stellar populations? How do galaxies and the intergalactic medium interact? How did planets and smaller bodies in our own solar system form and evolve?

AMTD uses science-driven systems engineering to derive engineering specifications directly traceable to science requirements, and mature technologies necessary to achieve those specifications - with an emphasis on solutions that are affordable, practical, and reduce risk. Because future launch capabilities or external technology advances are unpredictable, AMTD is pursuing multiple design paths to provide the science community with options to enable either large-aperture monolithic or segmented mirrors with clear engineering metrics. AMTD's demonstrated deepcore manufacturing method enables 4-m class mirrors with 20-30\% lower cost and risk. The design tools we have developed increase speed, resulting in reduced cost of trade studies. In addition, the integrated modeling tools we have developed enable better definition of system and component engineering specifications.

\section{Accomplishments}

AMTD-1 matured six key technologies required to make an integrated primary mirror assembly (PMA) for a large aperture UVOIR space telescope: (1) Large-Aperture, Low-Areal Density, High-Stiffness Mirror Substrates; (2) Support System; (3) Mid/High-Spatial Frequency Figure Error; (4) Segment Edges; (5) Segment-to-Segment Gap Phasing; and (6) Integrated Model Validation

AMTD-1's key accomplishment was the successful demonstration the new 5-layer 'stack \& fuse' process for fabricating deep-core mirror substrates. Using this new process, a $43 \mathrm{~cm}$ diameter 'cut-out' of a 4 m diameter, 40 -cm thick, $<45 \mathrm{~kg} / \mathrm{m} 2$ mirror substrate was fabricated (Figure 1). This demonstrated that the technology can make mirrors with sufficient thickness for a mechanically ultra-stable $4 \mathrm{~m}$ to $8 \mathrm{~m}$ class mirror.

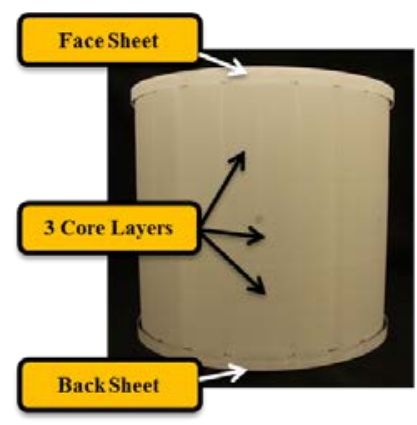

Post-Fusion Side View 3 Core Layers and Vent Hole Visible

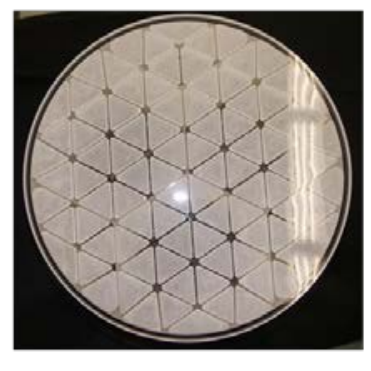

Post-Fusion Top View

Pocket Milled Faceplate

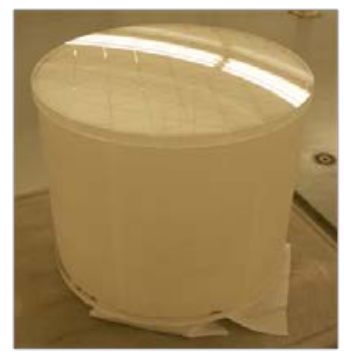

Post Slump:

2.5 meter Radius of Curvature

Fig. 1. Deep core mirror attributes and at different processing stages. 
AMTD-2 continued maturing three technologies needed to enable large monolithic (4- to 8-m) and segmented (8- to 16-m) UVOIR space telescopes: 1) Large-Aperture, Low-Areal Density, 2) High-Stiffness Substrates; Support System: to ensure launch survival and on-orbit stress-free deployment; and, 3) Integrated Model Validation: since on-orbit performance depends on mechanical and thermal stability and compliance cannot be $100 \%$ tested (because large telescopes are not stiff enough to test), validated models are required.

AMTD-2 successfully low-temperature-fused/low-temperature-slumped a 1.5-meter diameter ULEC mirror that is a 1/3rd scale model of a 4-meter mirror (Figure 2).
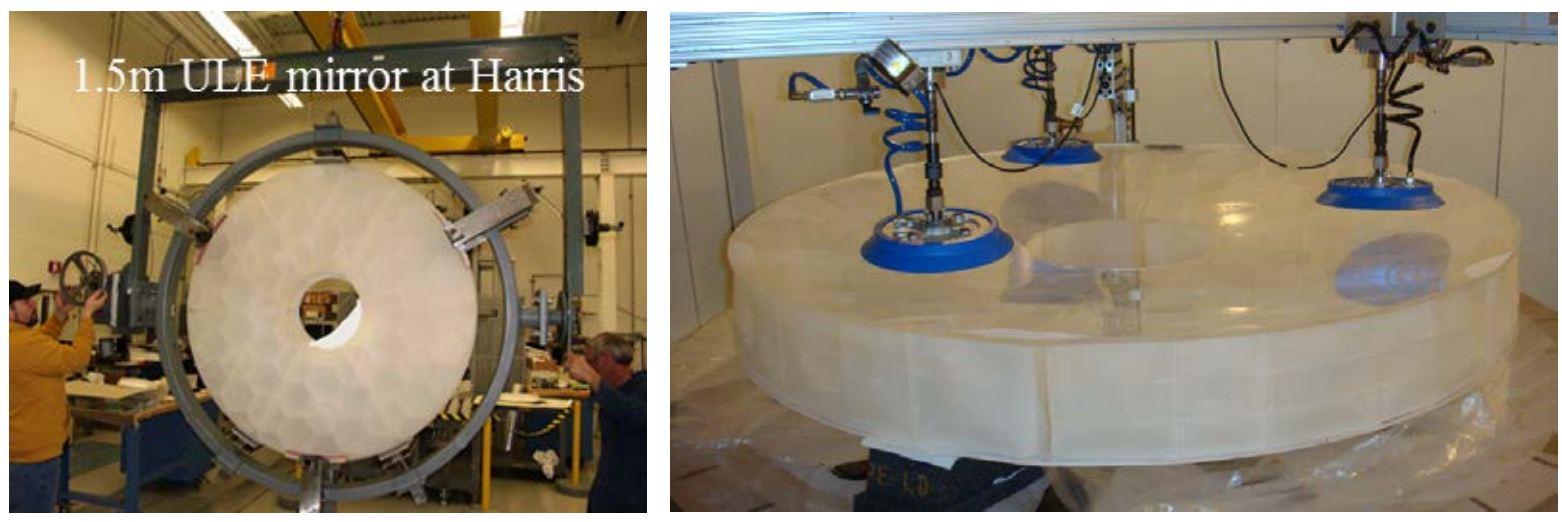

Figure 2: 1.5 meter diameter (1/3 $3^{\text {rd }}$ scale of 4-meter) LTF/LTS ULE@ mirror

Additionally, AMTD-2 characterized the cryogenic figure change of a 1.2-meter Extreme-Lightweight Zerodur ${ }^{\circledR}$ mirror (Figure 3) from ambient to 230K. To correlate test results with a performance model, the mirror was instrumented with multiple thermal sensors and its front surface temperature monitored with a thermal camera. The 1.5m ULE@ mirror will characterize in spring 2017.
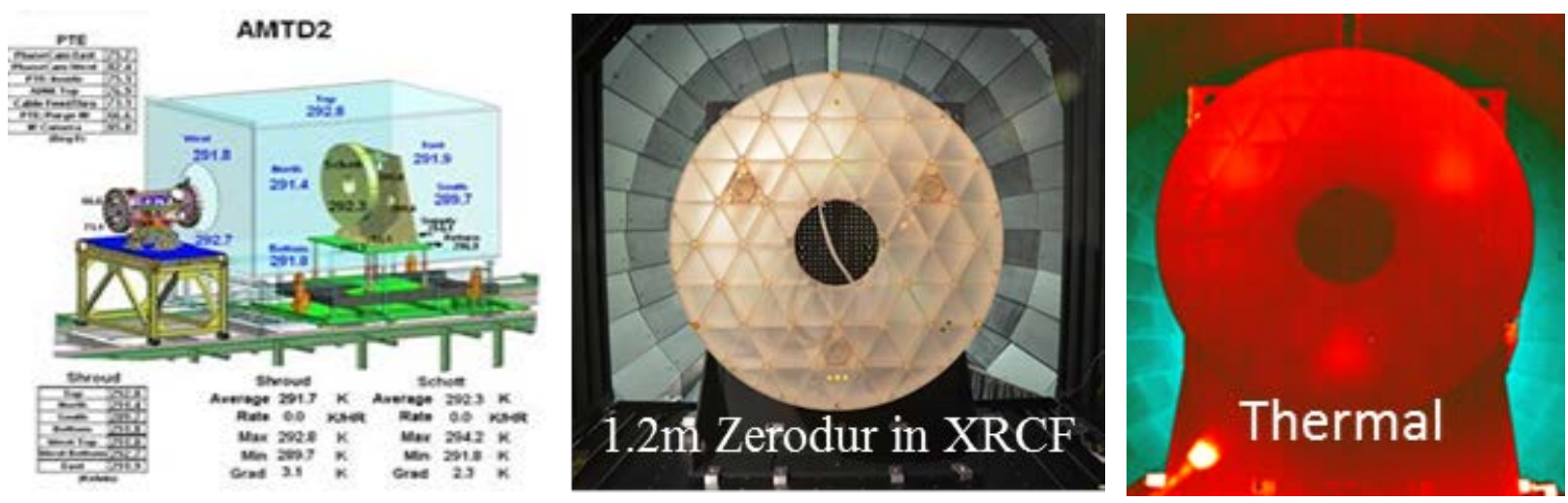

Figure 3: Static Thermal Test Configuration 1.5 meter diameter (1/3 ${ }^{\text {rd }}$ scale of 4-meter) LTF/LTS ULE@ mirror

Finally, the Zerodur ${ }^{\circledR}$ test results and performance model were correlated (Figure 4).
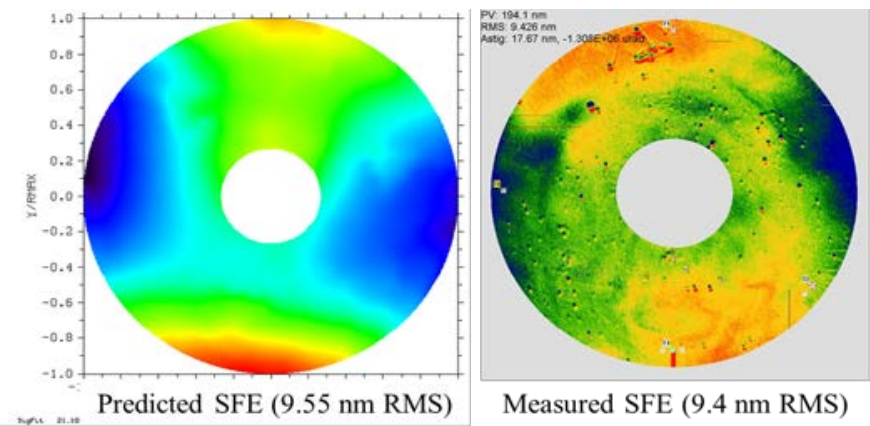

Figure 4: Predicted Thermal Deformation assuming 5 ppb CTE homogeinity and Measured Deformation. 


\section{AMTD Publications}

Stahl, H. Philip, “Advanced mirror technology development (AMTD) project: overview and year four accomplishments,” Proc. SPIE 9912, Advances in Optical and Mechanical Technology for Telescopes and Instrumentation II, (22 July 2016); doi:10.1117/12.2234082.

Stahl, H. Philip, “Overview and accomplishments of advanced mirror technology development phase 2 (AMTD-2) project”, Proc. SPIE. 9602, UV/Optical/IR Space Telescopes and Instruments: Innovative Technologies and Concepts VII, 960208. (September 22, 2015) doi: 10.1117/12.2186195.

Stahl, Mark T., H. Philip Stahl, and Stuart B. Shaklan "Preliminary analysis of effect of random segment errors on coronagraph performance”, Proc. SPIE. 9605, Techniques and Instrumentation for Detection of Exoplanets VII, 96050P. (September 24, 2015) doi: 10.1117/12.2190160.

Brooks, Thomas, H. P. Stahl, William R. Arnold, “Advanced Mirror Technology Development (AMTD) thermal trade studies”, Proc. SPIE. 9577, Optical Modeling and Performance Predictions VII, 957703. (September 23, 2015) doi: 10.1117/12.2188371

Egerman, et. al., "Status of the Advanced Mirror Technology Development (AMTD) phase 2 1.5m ULE mirror", Proc. SPIE. 9575, Optical Manufacturing and Testing XI, 95750L. (August 27, 2015) doi: 10.1117/12.2188566

Gersh-Range, Jessica; William R. Arnold, Sr.; H. Philip Stahl, "Edgewise connectivity: an approach to improving segmented primary mirror performance”, Journal of Astronomical Telescope and Instrument Systems, (2014) doi: 10.1117/1.JATIS.1.1.014002

Gersh-Range, Jessica; William R. Arnold, Sr.; David Lehner; H. Philip Stahl, "Flux-pinning mechanisms for improving cryogenic segmented mirror performance”, Journal of Astronomical Telescope and Instrument Systems, (2014) doi: 10.1117/1.JATIS.1.1.014001

Stahl, H. Philip; Marc Postman; Laura Abplanalp; William Arnold; Carl Blaurock; Robert Egerman; Gary Mosier, "Advanced mirror technology development (AMTD) project: 2.5 year status”. Proc. SPIE. 9143, Space Telescopes and Instrumentation 2014: Optical, Infrared, and Millimeter Wave, 91431S. (August 02, 2014) doi: 10.1117/12.2054765

Stahl, H. Philip; Marc Postman; Gary Mosier; W. Scott Smith; Carl Blaurock; Kong Ha; Christopher C. Stark, “AMTD: update of engineering specifications derived from science requirements for future UVOIR space telescopes”, Proc. SPIE. 9143, Space Telescopes and Instrumentation 2014: Optical, Infrared, and Millimeter Wave, 91431T. (August 02, 2014) doi: $10.1117 / 12.2054766$

Matthews, Gary W.; Robert Egerman; Steven P. Maffett; H. Philip Stahl; Ron Eng; Michael R. Effinger, "The development of stacked core technology for the fabrication of deep lightweight UV-quality space mirrors”, Proc. SPIE. 9143, Space Telescopes and Instrumentation 2014: Optical, Infrared, and Millimeter Wave, 91431U. (August 28, 2014) doi: 10.1117/12.2055284

Stahl, H. Philip, Marc Postman, Laura Abplanalp, William Arnold, Ron Eng, Anand Sivaramakrishnan "Overview and recent accomplishments of the advanced mirror technology development (AMTD) for large aperture UVOIR space telescopes project”, Proc. SPIE 8860, 2013, DOI: 10.1117/12.2022362

Stahl, H. Philip, Marc Postman and W. Scott Smith, "Engineering specifications for large aperture UVO space telescopes derived from science requirements”, Proc. SPIE 8860, 2013, DOI: 10.1117/12.2024480

Matthews, Gary W., et. al., "Development of stacked core technology for fabrication of deep lightweight UV-quality space mirrors”, Proc. SPIE 8838, 2013, DOI: 10.1117/12.2024630

Matthews, Gary W., et. al., "Processing of a stacked core mirror for UV applications”, Proc. SPIE 8837, 2013, DOI: $10.1117 / 12.2024644$

Eng, Ron, et. al., "Cryogenic optical performance of a lightweighted mirror assembly for future space astronomical telescopes: correlating optical test results and thermal optical model”, Proc. SPIE 8837, 2013, DOI: 10.1117/12.2025393

Arnold, William R., Matthew Fitzgerald, Rubin Jaca Rosa, and H. Philip Stahl, "Next-generation lightweight mirror modeling software”, Proc. SPIE 8836, 2013, DOI: 10.1117/12.2023509

Arnold, William R., Ryan M. Bevan and H. Philip Stahl, “Integration of mirror design with suspension system using NASA's new mirror modeling software”, Proc. SPIE 8836, 2013, DOI: 10.1117/12.2023512

Stahl, Mark T. and H. Philip Stahl, “Incorporating skew into RMS surface roughness probability distributions”, Proc. SPIE 8860, 2013, DOI: $10.1117 / 12.2024883$ 